\title{
Glycolytic competence in gastric adenocarcinomas negatively impacts survival outcomes of patients treated with salvage paclitaxel-ramucirumab
}

\author{
Annamaria Ruzzo ${ }^{1}$ (D) Francesco Graziano ${ }^{2}$. Irene Bagaloni ${ }^{1} \cdot$ Maria Di Bartolomeo $^{3} \cdot$ Michele Prisciandaro $^{3}$. \\ Giuseppe Aprile ${ }^{4}$ Elena Ongaro ${ }^{5}$ - Bruno Vincenzi ${ }^{6}$ - Giuseppe Perrone ${ }^{6}$. Daniele Santini ${ }^{6}$. Lorenzo Fornaro ${ }^{7}$. \\ Caterina Vivaldi ${ }^{7}$. Gianluca Tomasello ${ }^{8}$. Fotios Loupakis ${ }^{9} \cdot$ Sara Lonardi $^{9}$. Matteo Fassan ${ }^{10}$ • Michele Valmasoni ${ }^{11}$. \\ Donatella Sarti ${ }^{2} \cdot$ Paola Lorenzini ${ }^{2} \cdot$ Vincenzo Catalano $^{2} \cdot$ Renato Bisonni $^{12} \cdot$ Michela Del Prete $^{12} \cdot$ Guido Collina $^{13}$. \\ Mauro Magnani ${ }^{1}$
}

Received: 25 February 2020 / Accepted: 23 April 2020 / Published online: 5 May 2020

(c) The Author(s) 2020

\begin{abstract}
Introduction For energy production, cancer cells maintain a high rate of glycolysis instead of oxidative phosphorylation converting glucose into lactic acid. This metabolic shift is useful to survive in unfavorable microenvironments. We investigated whether a positive glycolytic profile (PGP) in gastric adenocarcinomas may be associated with unfavorable outcomes under an anticancer systemic therapy, including the anti-angiogenic ramucirumab.

Materials and methods Normal mucosa (NM) and primary tumor (PT) of 40 metastatic gastric adenocarcinomas patients who received second-line paclitaxel-ramucirumab (PR) were analyzed for mRNA expression of the following genes: $H K$ - 1 , $H K-2, P K M-2, L D H-A$, and GLUT-1. Patients were categorized with PGP when at least a doubling of mRNA expression (PT vs. NM) in all glycolytic core enzymes (HK-1 or HK-2, PKM-2, LDH-A) was observed. PGP was also related to TP53 mutational status.

Results Mean $L D H-A, H K-2, P K M-2$ mRNA expression levels were significantly higher in PT compared with NM. 18 patients were classified as PGP, which was associated with significantly worse progression-free and overall survival times. No significant association was observed between PGP and clinical-pathologic features, including TP53 positive mutational status, in 28 samples.

Conclusions Glycolytic proficiency may negatively affect survival outcomes of metastatic gastric cancer patients treated with PR systemic therapy. TP53 mutational status alone does not seem to explain such a metabolic shift.
\end{abstract}

Keywords Glycolysis · Warburg effect · Ramucirumab · Paclitaxel · Angiogenesis

Annamaria Ruzzo and Francesco Graziano have contributed equally.

Electronic supplementary material The online version of this article (https://doi.org/10.1007/s10120-020-01078-0) contains supplementary material, which is available to authorized users.

Annamaria Ruzzo

annamaria.ruzzo@uniurb.it

Francesco Graziano

francesco.graziano@ospedaliriunitimarchenord.it

Extended author information available on the last page of the article

\section{Background}

For energy production under aerobic conditions, normal cells generally transform glucose into carbonic anhydride by means of oxidative phosphorylation. Conversely, glycolysis with ultimate production of lactate is predominant in invasive cancer cells, even in the presence of sufficient levels of oxygen [1]. Although the incomplete oxidation of glucose to lactate yields only $5 \%$ of the energy available from glucose, this apparently senseless waste of glucose actually constitutes a survival advantage in rapidly proliferating cells. In fact, it makes them insensitive to transient or permanent hypoxic conditions, it contributes to the production of nucleosides and amino acids, and it constitutes a very rapid way 
to produce energy $[1,2]$. Furthermore, lactate is not just a waste product of this process; on the contrary, it promotes tumor invasion by favouring cell migration, angiogenesis, immune escape and radioresistance [3]. This metabolic shift is promoted by the over-expression of the key effectors of the glycolytic pathway $[4,5]$, including specific membrane glucose transporters (GLUT-1), and enzymes involved in the promotion of each single step of the glycolytic cascade (Fig. 1). The over-expressed enzymes themselves are subject to selection with some isoforms more frequently represented in tumor cells $[4,5]$.

In a previous study in metastatic colorectal cancer [6], we found mRNA tumor overexpression of GLUT- 1 and the glycolytic genes hexokinase $1(H K-1)$ and $2(H K-2)$, pyruvate kinase isoform $2(P K M-2)$ and lactate dehydrogenase isoform A $(L D H-A)$. In the subset of patients treated with anti-angiogenic bevacizumab, the glycolytic profile showed signals of detrimental association with survival outcomes
[6]. In fact, clones can be selected that have the ability to survive anti-angiogenic therapy-induced hypoxia, and the selection of hypoxia -resistant clones can also be observed with VEGF (vascular epithelial growth factor) receptor-1 and -2 inhibition $[7,8]$. These clones require fewer proangiogenic factors to promote their growth and proliferation and they possess phenotypic properties allowing them to overcome the lack of energy and nutrients supply [7, 8]. Most relevant, metabolic adaptation with a glycolytic shift may not be a simple prognostic biomarker $[9,10]$, but it may indicate innovative treatment strategies and novel drug targets in anti-cancer therapy [11-13].

This background prompted us to plan a novel study for evaluating the possible negative impact of the up-regulated glycolytic profile in patients exposed to anti-angiogenics. We focused on the paclitaxel-ramucirumab (PR) association for second-line therapy in metastatic gastric cancer. Ramucirumab is a recombinant human immunoglobulin G1

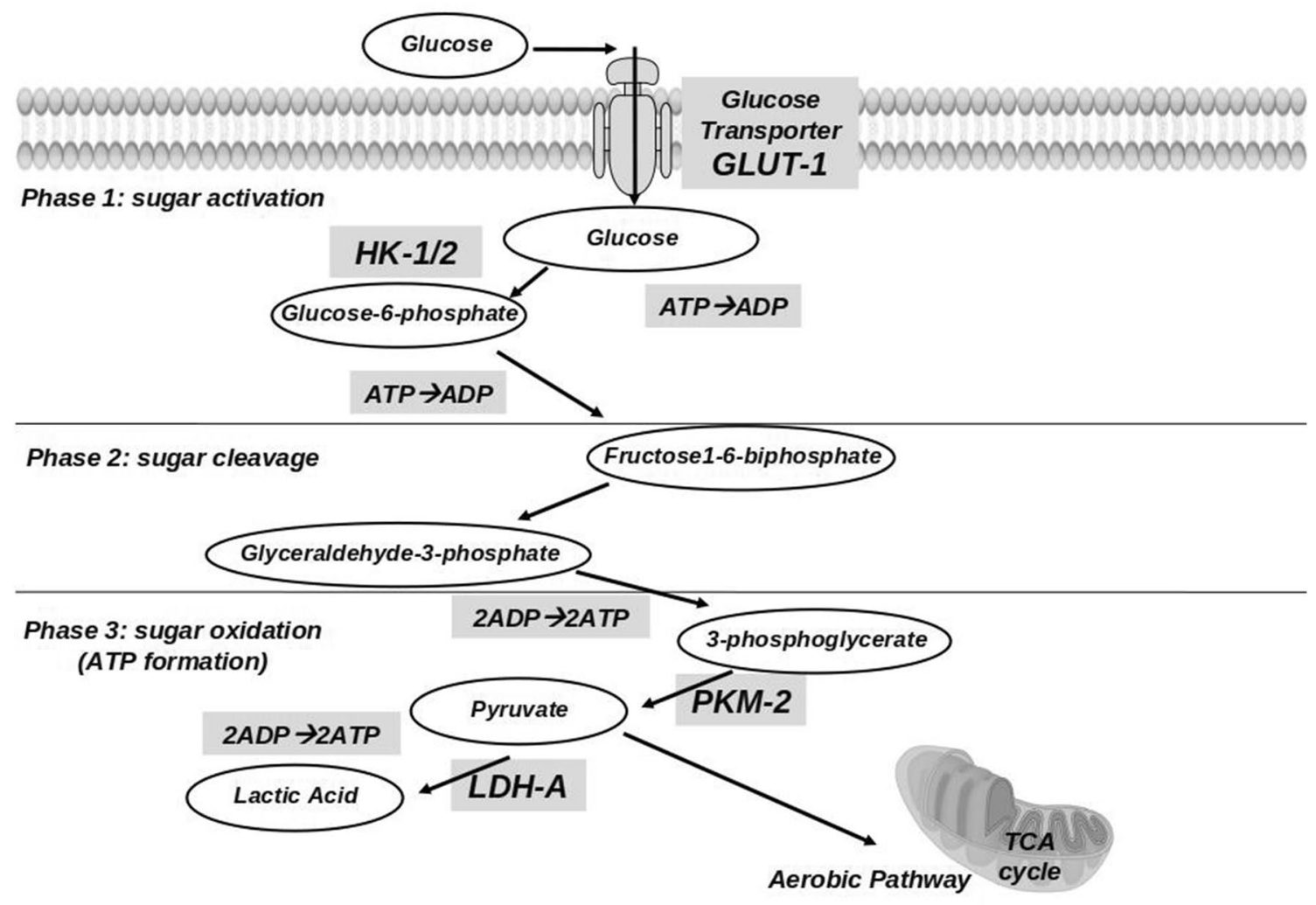

Fig. 1 Schematic representation of the three main steps (sugar activation, cleavage and oxidation) in the glycolysis pathway. Glucose transport-1 (GLUT-1) mediates the internalization of glucose across the plasma membrane. Hexokinase (HK-1 and HK-2) transfer one phosphate group from ATP to glucose, yielding glucose-6-phosphate (G6P). G6P may be shunted into the non-oxidative arm of the pentose phosphate pathway (PPP), otherwise it is converted through the intermediate reaction of glycolysis to 3-phosphoglycerate. Pyruvate kinase (PKM-2) catalyzes the transfer of a phosphate group from 3-phosphoglycerate to ADP, to give pyruvate and ATP. In the pres- ence of oxygen, cells completely oxidize most of that pyruvate in the mitochondria to $\mathrm{CO}_{2}$ during the process of oxidative phosphorylation in the tricarboxylic acid (TCA) cycle. When oxygen is limited, cells can redirect the pyruvate generated by glycolysis away from mitochondrial oxidative phosphorylation by generating lactate (anaerobic glycolysis). Lactate dehydrogenase isoform A (LDH-A) catalyzes the reversible conversion of pyruvate to lactate with the simultaneous oxidation of the cofactor $\mathrm{NADH}$ to $\mathrm{NAD}^{+}$. Warburg observed that cancer cells tend to convert most glucose to lactate regardless of whether oxygen is present (aerobic glycolysis) 
(IgG1) neutralizing monoclonal antibody specific for VEGF receptor-2 that prevents ligand binding and receptor-mediated pathway activation in endothelial cells. It is approved as a single agent, or in combination with paclitaxel, for the treatment of patients with advanced or metastatic gastric or gastroesophageal junction cancer with disease progression or after prior fluoropyrimidine or platinum-containing chemotherapy [14].

Interestingly, recent studies have revealed novel functions of the TP53 tumor-suppressor gene including the regulation of glycolysis [15]. In pre-clinical models, the p53 protein has been shown to repress glycolysis through multiple mechanisms. In particular, p53 transcriptionally represses the expression of glucose transporters and it was found to down-regulate the HK-2 and PKM-2 glycolytic enzymes. Also, p53 induces the expression of TIGAR (TP53-induced glycolysis and apoptosis regulator), which decreases the intracellular concentrations of fructose-2,6-bisphosphate, and thus reduces glycolysis and diverts glucose catabolism to the pentose phosphate pathway $[16,17]$. The TP53 gene is frequently mutated in gastric adenocarcinomas, and unlike many other tumor suppressors, the majority of TP53 mutations are missense, which usually leads to the production of the full-length mutant protein [18]. Also, it has been welldocumented that some mutant p53 proteins not only lose the tumor-suppressive function, but they gain new oncogenic functions as a result of the so-called "gain-of-function" TP53 mutations [18, 19]. In this study, we also devoted an ancillary analysis to TP53 mutational status to evaluate whether signals of p53 regulation of the glycolytic shift are detectable in vivo.

\section{Methods}

Italian institutions involved in the RAMos retrospective study [20] were asked to participate in the present study. To evaluate the results of a translational analysis in a homogeneous population of patients, this retrospective investigation focused on patients treated with the combination of ramucirumab and paclitaxel only. Therefore, patients were required to be treated with ramucirumab $8 \mathrm{mg} / \mathrm{kg}$ on days 1 and 15 , with paclitaxel $80 \mathrm{mg} / \mathrm{m}^{2}$ on days 1,8 and 15 , and both intravenously every 28 days. Availability of paired tissues of the primary tumor (PT) and normal mucosa (NM) was required for study inclusion. To characterize the glycolytic shift in cancer cells, mRNA over-expression of key enzymes in the three main phases of the glycolytic pathway were studied (Fig. 1). The relationship between levels of the mRNA and survival outcomes was assessed. All patient information and pathology material were collected under a protocol approved by the Regional Ethical Committee.
Patients were asked to provide additional written informed consent (see supplementary information file).

\section{Samples and nucleic acids extraction}

Four to six $10-\mu \mathrm{m}$ sections from formalin-fixed, paraffinembedded (FFPE) specimens were obtained from PT and matched NM. The sample preparation protocol expressly indicated the acquisition of NM samples from surgical or biopsy blocks with accurate identification of healthy gastric mucosa. These sections had to be distinct from those prepared for tumor sampling thus excluding proximity to tumor infiltration. Before cutting sections for total nucleic acids isolation, an additional slide was prepared for hematoxylin-eosin staining and the pathologists identified representative areas with an almost complete representation of tumor infiltration. For each enrolled patient, total DNA and RNA were extracted from PT and matched NM. Both tissues were micro-dissected and placed in a $1.5 \mathrm{ml}$ reaction tube containing $1 \mathrm{ml}$ xylene to remove paraffin. DNA and RNA were extracted using the RecoverAll ${ }^{\mathrm{TM}}$ Multi-Sample RNA/ DNA Isolation Workflow (Invitrogen ${ }^{\mathrm{TM}}$, CA, USA) according to the manufacturer's instructions. DNA and RNA concentration and purity were measured using the NanoDrop 1000 spectrophotometer (Nanodrop Technologies, Rockland, DE, USA).

\section{CDNA synthesis and quantitative real-time PCR (RT-qPCR)}

The SuperScript ${ }^{\mathrm{TM}}$ VILO $^{\mathrm{TM}}$ cDNA Synthesis kit (Invitrogen $^{\mathrm{TM}}$, CA, USA) was used to generate first-strand cDNAs from $1 \mu \mathrm{g}$ of total RNA according to the manufacturer's instructions. The products were diluted to obtain a final concentration of $10 \mathrm{ng} / \mu \mathrm{l}$ of reverse-transcribed mRNA. Quantitative real-time PCR (RT-qPCR) was performed to analyze the mRNA expression levels of the five candidate genes ( $H K$ 1, HK-2, PKM-2, LDH-A, and GLUT-1) and two reference genes (B2M and GUSB), as previously reported [6]. Briefly, RT-qPCR was carried out using TaqMan Gene Expression Assay and TaqMan Gene Expression Mastermix (Applied Biosystems, Foster City, CA, USA) following the manufacturer's protocol. All reactions were performed in triplicate and each PCR run included a no-template control. The RTqPCR Ct values (the average value of the triplicates) were converted in $\mathrm{Cy} 0$ by a tool for accurate and precise quantification [21] and the relative mRNA expression of each target was calculated as $\Delta \mathrm{Cy} 0=\mathrm{Cy} 0_{\text {(target gene) }}-\mathrm{Cy} 0_{\text {(reference gene). }}$. In this analysis, a higher mRNA expression level corresponds to a smaller $\Delta \mathrm{Cy} 0$ value.

Subsequently, the $2^{-\Delta \Delta \mathrm{Cy} 0}$ method was used to express the $n$-fold differential expression (fold change) of each candidate gene between the tumor sample and the normal 
counterpart. Fold change $\geq 2$ indicates a doubling in the mRNA content and it was adopted as a threshold for differential RNA expression in microarray and drug induction studies [22-25].

\section{Amplicons library preparation and next-generation sequencing (NGS) for TP53 analysis}

A custom panel (IAD_119861) including the TP53 coding and the relative UTR regions was designed using the Ion AmpliSeq ${ }^{\mathrm{TM}}$ Designer software (Thermo Fisher, Foster City, CA). The panel was made up of 35 amplicons and ensured $82 \%$ of gene coverage. The Ion AmpliSeq Library Kit Plus was used to prepare the libraries according to the manufacturer's instructions. Libraries were generated using $40 \mathrm{ng}$ of tumor DNA and indexed using the Ion Xpress Barcode Adapter Kit. Library purification was carried out using the AMPure ${ }^{\mathrm{TM}}$ XP Reagent (Beckman Coulture, CA, USA) on the DynaMagTM-2 Magnet. Qubit ${ }^{\text {TM }} 4$ Fluorometer (Invitrogen ${ }^{\mathrm{TM}}$, , CA, USA) was used to quantify amplicons libraries. After dilution of all samples at $100 \mathrm{pM}$, libraries were pooled for emulsion PCR on the Ion OneTouch ${ }^{\mathrm{TM}} 2$ instrument, using the Ion S5 ${ }^{\mathrm{TM}}$ Template OT2 kit, according to the manufacturer's instructions. The Ion Sphere ${ }^{\mathrm{TM}}$ Particles were enriched using the Ion OneTouch ${ }^{\mathrm{TM}}$ Enrichment System and the template was sequenced on the Ion Torrent S5 platform using the Ion $540^{\mathrm{TM}}$ Chip following the manufacturer's instruction. All of these instruments and reagents were supplied by Thermo Fisher (Foster City, CA). Read alignment was performed using hg 19 (GRCh37) as the reference genome.

Variant call files were generated by the Variant Caller v. 5 plugin pre-installed in the Torrent Suite and analyzed with the Ion Reporter ${ }^{\mathrm{TM}}$ software (Thermo Fisher, Foster City, CA). BAM files were also manually checked on IGV (Integrative Genomics Viewer) [26]. Mutations were categorized as "disruptive" $\left(T P 53_{\mathrm{D}}\right)$ or "non-disruptive" $\left(T P 53_{\mathrm{ND}}\right)$ according to the classification of Poeta et al. [27]. Mutations were also classified as "gain-of-function" (TP53 $\left.3_{\mathrm{GOF}}\right)$ if reported in current databases from the review of available studies in which a clear gain-of-function effect was shown [19].

\section{Statistical analysis}

mRNA expression levels were reported as $\Delta \mathrm{Cy} 0$ values with means and standard deviations; group differences were compared using two-sample t- and Wilcoxon tests. Significant associations for each gene were required to be detectable with both reference genes. Contingency tables were analyzed by the Fisher's exact test. Statistical significance was defined as $p<0.05$.
For the purpose of clinical associations, fold change results produced by the $2^{-\Delta \Delta C y 0}$ method were adopted. Cases were defined as having a positive glycolytic profile (PGP) when fold changes $\geq 2$ (PT vs. NM) were present in all glycolytic core genes: $H K-1$ or $H K-2, P K M-2, L D H-A$, and GLUT-1. The remaining cases were categorized as having a negative glycolytic profile (NGP).

The primary end-point was overall survival (OS), which was compared between PGP and NGP groups to assess the possible clinical impact of glycolytic proficiency. OS was calculated from the date of the first cycle of second-line PR therapy to the earliest of date of death or last followup. Progression-free survival (PFS) was the secondary endpoint, defined as the time from the date of the first cycle of second-line PR therapy to the earlier of disease progression or death, or last follow-up.

The Kaplan-Meier method was used to estimate survival curves and the log-rank test was used to compare survival times between PGP and NGP groups. A multivariable Cox proportional hazards model was then used to adjust for clinical and pathologic features. Two-sided $p$ values $95 \%$ confidence intervals (CI) were reported. A $p$ value $<0.05$ was considered statistically significant.

Statistical analyses were performed using MedCalc for Windows, version 15.0 (MedCalc Software, Ostend, Belgium).

\section{Results}

Forty consecutive patients who underwent PR second-line systemic therapy and had paired archival tissue samples of the PT and matched NM were included from eight Italian institutions. All patients received first-line chemotherapy with a platinum derivate (cisplatin or oxaliplatin) plus fluoropyrimidines. In the second-line setting, patients underwent PR between September 2015 and September 2018 (Table 1). The results of second-line therapy in this cohort of patients parallel findings in the RAMos study [20]. The overall response rate was $17.5 \%$, with 7 partial responses in the 40 patients. In the whole group, the median OS was 7.8 months (95\% CI 4.5 to 8.6 months) and the median PFS was 3.8 months (95\% CI 3.2 to 4.6 months).

\section{Expression analyses}

As shown in Fig. 2, statistically significant differences in mRNA expression levels were detected comparing $\triangle \mathrm{Cy} 0$ values between $\mathrm{PT}$ tissues and matched NM for $H K-2, P K M$ 2, GLUT-1 and LDH-A.

The ranking of fold change analysis is reported in Fig. 3. Fold change $\geq 2$ was detected in 19 cases for GLUT-1, in 19 cases for $L D H-A$, in 19 cases for $P K M-2$, in 13 cases 
Table 1 Characteristics and distribution of the 40 patients according to the glycolytic profile

\begin{tabular}{|c|c|c|c|c|}
\hline \multirow[t]{2}{*}{ Variable } & \multicolumn{3}{|c|}{ Number of patients (\%) } & \multirow[b]{2}{*}{$p$ value } \\
\hline & Total & PGP & NGP & \\
\hline \multicolumn{5}{|l|}{ Age } \\
\hline$>70$ years & $29(72.5)$ & $11(61)$ & $18(82)$ & \multirow[t]{2}{*}{0.7} \\
\hline$<70$ years & $11(27.5)$ & $7(39)$ & $4(18)$ & \\
\hline \multicolumn{5}{|l|}{ Gender } \\
\hline Male & $18(45)$ & $7(39)$ & $11(50)$ & \multirow[t]{2}{*}{0.5} \\
\hline Female & $22(55)$ & $11(61)$ & $11(50)$ & \\
\hline \multicolumn{5}{|l|}{ PFS1 time } \\
\hline$>6$ months & $26(65)$ & $13(72)$ & $13(59)$ & \multirow[t]{2}{*}{0.5} \\
\hline$<6$ months & $14(35)$ & $5(28)$ & $9(41)$ & \\
\hline \multicolumn{5}{|c|}{$\begin{array}{l}\text { Number of metastatic } \\
\text { sites }\end{array}$} \\
\hline 1 & $21(52.5)$ & $6(33)$ & $15(68)$ & \multirow[t]{2}{*}{0.05} \\
\hline$>2$ & $19(47.5)$ & $12(67)$ & $7(32)$ & \\
\hline \multicolumn{5}{|c|}{ Peritoneum involvement } \\
\hline Positive & $21(52.5)$ & $10(55)$ & $11(50)$ & \multirow[t]{2}{*}{0.7} \\
\hline Negative & $19(47.5)$ & $8(45)$ & $11(50)$ & \\
\hline \multicolumn{5}{|l|}{ ECOG PS } \\
\hline 0 & $22(55)$ & $10(55)$ & $12(54.5)$ & \multirow[t]{2}{*}{0.5} \\
\hline $1-2$ & $18(45)$ & $8(45)$ & $10(45.5)$ & \\
\hline \multicolumn{5}{|c|}{ Lauren's histology } \\
\hline Intestinal & $26(65)$ & $14(78)$ & $12(54.5) 0.9$ & \\
\hline Diffuse & $14(45)$ & $4(22)$ & $10(45.5)$ & \\
\hline \multicolumn{5}{|l|}{ Grading } \\
\hline $1-2$ & $18(45)$ & $10(55)$ & $8(36)$ & \multirow[t]{2}{*}{0.3} \\
\hline 3 & $22(55)$ & $8(45)$ & $14(64)$ & \\
\hline \multicolumn{5}{|c|}{ Primary tumor resected } \\
\hline Yes & $18(45)$ & $7(39)$ & $11(50)$ & \multirow[t]{2}{*}{0.1} \\
\hline No & $22(55)$ & $11(61)$ & $11(50)$ & \\
\hline \multicolumn{5}{|c|}{ Primary tumor site } \\
\hline Cardia & $9(22.5)$ & $5(28)$ & $4(18)$ & \multirow[t]{2}{*}{0.7} \\
\hline Non-cardia & $21(77.5)$ & $13(72)$ & $18(82)$ & \\
\hline
\end{tabular}

$P G P$ positive glycolytic profile, $N G P$ negative glycolytic profile, PFS1 progression-free survival to first-line chemotherapy, ECOG PS Eastern Cooperative Group Performance Status

for $H K$ - 2 and in 16 cases for $H K$ - 1 . Eighteen cases showed synchronous fold change $\geq 2$ in one of the two hexokinases (HK-2 or $H K-1), P K M-2, L D H-A$, and they composed the PGP group. Notably, all the PGP cases showed also fold change $\geq 2$ for GLUT-1. The remaining 22 cases composed the NGP group. Intriguingly, a clear-cut distribution of fold change $\geq 2$ expression levels in the four analyzed target genes seems to be present. In fact, except for case number 16 and 30, NGP group cases showed none or a single mRNA fold change $\geq 2$.

As shown in Table 1, the distribution of clinical and pathologic features of the 40 patients according to PGP and NGP status did not show statistically significant associations. A borderline $p$ value ( $p=0.05$ ) was observed between PGP and NGP groups for the number of metastatic sites, with a numerically greater prevalence of PGP patients having more extensive metastatic disease.

PGP status did not differentiate treatment responses to second-line therapy $(p>0.05)$. There were 2 partial response in the PGP group and 5 in the NGP group. Four patients had stable disease in the PGP group and 10 in the NGP group. Disease progression occurred in 12 patients in the PGP group and in 7 patients in the NGP group. The number of patients with partial response and stable disease in the disease-control rate (DCR) was statistically significantly different between the two groups with DCR in 15 and 6 patients in the NGP and PGP groups, respectively $(p=0.03)$.

\section{Expression analysis and TP53 status}

TP53 mutations in the coding region were found in 28 patients (70\%). All detected TP53 mutations were missense mutations described in the IARC database. Their characteristics are reported in Fig. 3, together with the distribution of cases according to glycolytic status. Eleven carriers of a TP53 mutation were in the PGP group and 17 were in the NGP group. Seven patients without TP53 mutations were in the PGP group and 5 in the NGP group. The association between TP53 mutation and glycolytic status was not statistically significant $(p=0.20)$. The TP53 missense mutations were classified as $T P 53_{\mathrm{ND}}$ in 7 cases, $T P 53_{\mathrm{D}}$ in 10 cases and $T P 53_{\mathrm{GOF}}$ in 11 cases. In particular, all but one of the $T P 53_{\mathrm{ND}}$ mutations occurred in the NGP group. Six of the $10 T P 53_{D}$ mutations were in the PGP group and 7 of the $11 T P 53_{\mathrm{GOF}}$ mutations were the NGP group $(p=0.1)$.

\section{Survival analysis}

In the OS analysis of second-line PR systemic therapy (see Fig. 4, Panel A), patients with a NGP showed statistically significant better survival than those with PGP: median OS of 8.9 months (95\% CI 7.8-10.7 months) vs. median OS of 4.1 months (95\% CI 3.5-5.3 months), respectively $(p=0.008)$. Similarly, glycolytic status showed a statistically significant impact on PFS (Fig. 4, panel B). Median PFS in NGP patients was 4.9 months (95\% CI 4.4-6.1 months) and median PFS in PGP patients was 2.0 months $(95 \% \mathrm{CI}$ $1.9-3.7$ months $)(p=0.02)$.

The results of the multivariable Cox regression analysis are shown in Table 2 . The glycolytic profile retained independent associations with PFS and OS, after controlling for other prognostic factors. In the OS analysis, adverse outcome was associated with PGP status, ECOG performance status 1-2, peritoneum involvement and the presence of the primary gastric tumor. 
Fig. 2 Box plot with standard deviation (SD) bars showing mRNA expression levels of the candidate genes in the primary tumor (T) and normal mucosa $(\mathrm{N})$. Data are presented as $\Delta \mathrm{Cy} 0$ values: the smaller the $\Delta \mathrm{Cy} 0$ value, the higher the expression

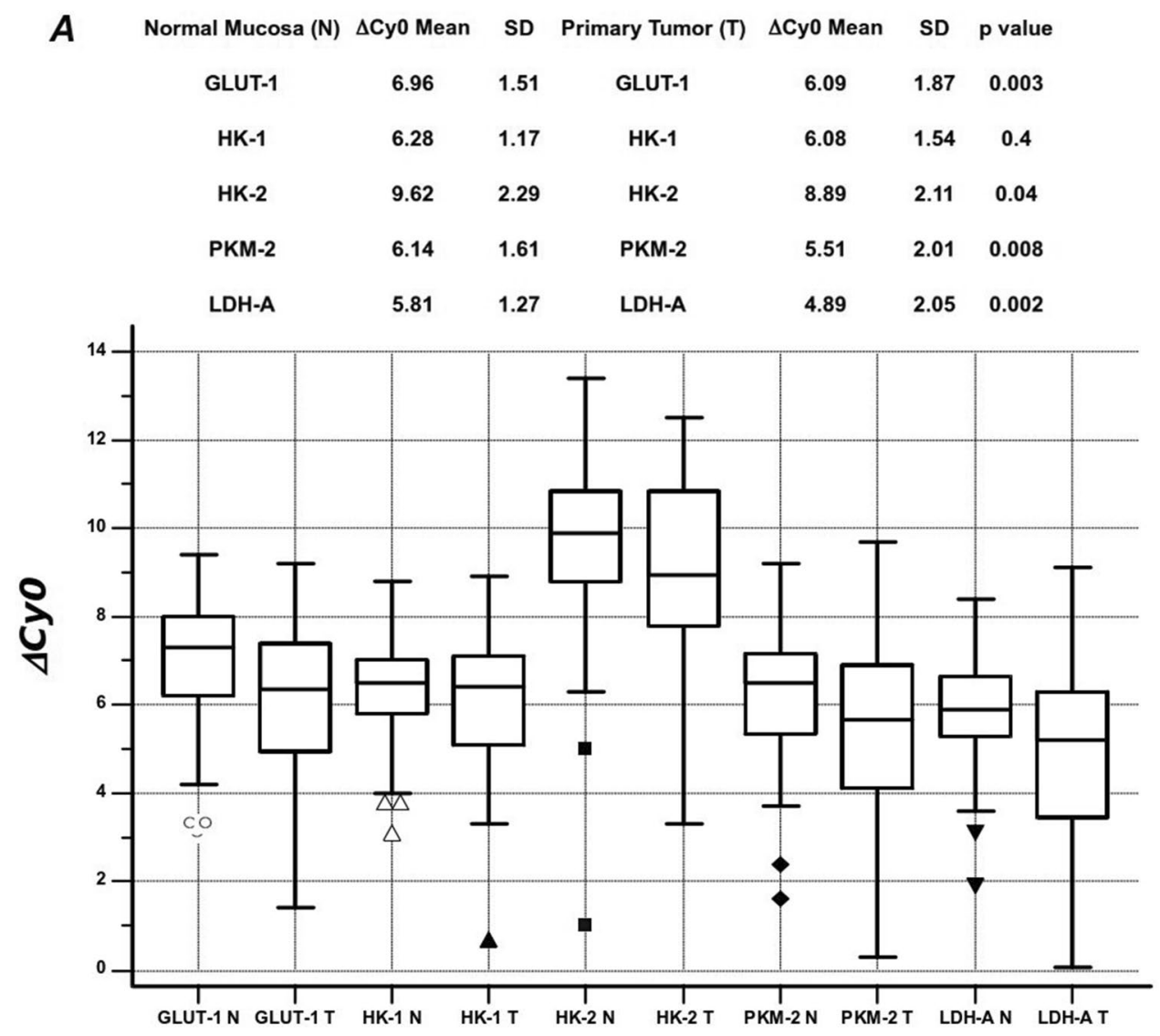

\section{Discussion}

Even after a decade from the FDA approval of the first anti-VEGF drug bevacizumab, resistance to anti-VEGF therapy remains a challenge in the treatment of cancer patients. Mechanisms of resistance are described as being intrinsic (preexisting) or adaptive (acquired), and they may explain why some tumors do not respond from the outset and why others progress after initial shrinkage. Redundancy of pro-angiogenic growth factors and activation of alternative angiogenic pathways have been investigated and considered as the prevalent mechanisms of resistance to anti-angiogenic compounds in cancer therapy $[8,9]$. In recent years, many pre-clinical and translational studies indicated that metabolic reprogramming with adaptive escape in response to a hypoxic tumor microenvironment may offer a novel and intriguing opportunity for explaining the failure of anti-angiogenic treatments in solid tumors. In particular, glycolysis is an essential metabolic pathway in the hypoxic adaptation for survival and tumor progression. In this perspective, tumors may develop early and/ or late resistance to anti-angiogenic agents when clones are more equipped for prompt and redundant metabolic changes (i.e., glycolytic shift) in a therapeutically induced hypoxic environment $[7,9]$.
In the past few years, translational clinical studies in cancer patients have addressed the putative clinical impact of glycolysis-related proteins and factors on prognosis, and there is mounting evidence that these features negatively affect survival outcomes [28]. The gastric cancer setting was analyzed in some of these studies. Findings showed significant associations between poor prognosis and more advanced stage disease or adverse histological features with up-regulated expression of glucose transporters [29-31] hexokinases [32-34] pyruvate kinases [34, 35], other enzymes involved in energy metabolism [36, 37], and lactate dehydrogenase [38-40]. To the best of our knowledge, this is the first study that analyzes a homogenous population of metastatic gastric cancer patients treated with a regimen that includes an anti-angiogenic compound. Moreover, we attempted an approach with multiple genes to determine a PGP rather than a single-component analysis.

A starting point for discussion is the characterization of the population of gastric cancer patients whose tumors displayed the positive glycolytic profile. Given the relatively early interest in the clinical impact of cancer metabolic features, there is a lack of standardized criteria and almost all studies investigated single glycolysis-related factors with different methods [28]. We formulated an approach which combined the biochemical principle of 


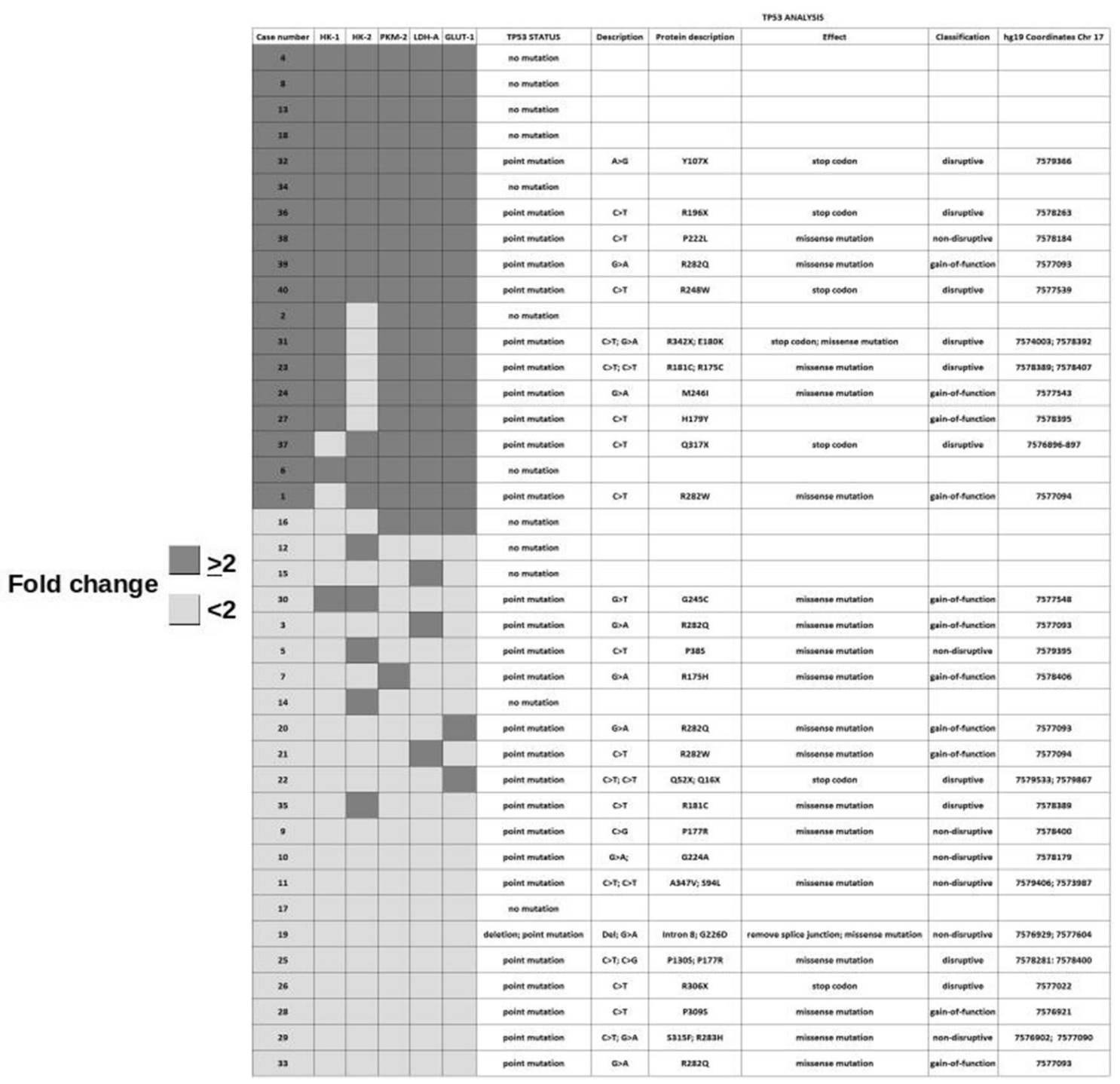

Fig. 3 Results of tumor profiling according to fold-change mRNA analysis. Cases were categorized as positive glycolytic profile (PGP) when fold-change $\geq 2$ simultaneously occurred in $H K-1$ or $H K$ -

the glycolytic cascade in its main enzymatic steps [41], together with a sensitive threshold for mRNA expression in vivo $[23,25]$. Fold-change is a very intuitive method to identify differentially expressed genes and it quantifies the change of expression levels. In fold-change analyses, 1.5 or 2 is often used as the cut-off to choose differentially expressed genes. The contemporary up-regulation of three key glycolytic enzymes coupled with fold-change $\geq 2$ in mRNA expression in cancer tissues compared with their normal counterpart is a fairly strict criterion to label a PGP case. In a comparative analysis between tissues, the quality of sampling is mandatory for reducing the risk of biases. For example, the result of a NGP status could be a falsenegative if sampling was made in deceptively healthy NM areas. In our study, pre-specified procedures for sampling
2, $P K M-2, L D H-A$ (dark grey squares). White squares denote the remaining cases with a negative glycolytic profile (NGP) because fold-change $<2$ or fold- change $\geq 2$ in only one or two mRNA and the involvement of expert pathologists in the selection of tissues should have minimized this risk. The observed differences in mRNA expression levels of target genes between NM and PT tissues would suggest an effective procedure to seize the presence of the glycolytic shift. We cannot rule out that an expression level analysis of each target gene in PT or NM tissues could be also predictive of clinical outcomes. However, to our opinion, this approach would be less informative on a global glycolytic shift and it could be done after identifying a cut-off level for each tested gene. We acknowledge that our criteria necessitate replication in independent studies and additional settings. Hopefully, they could lay the groundwork for a standardized determination of a glycolytic profile in translational cancer studies. 

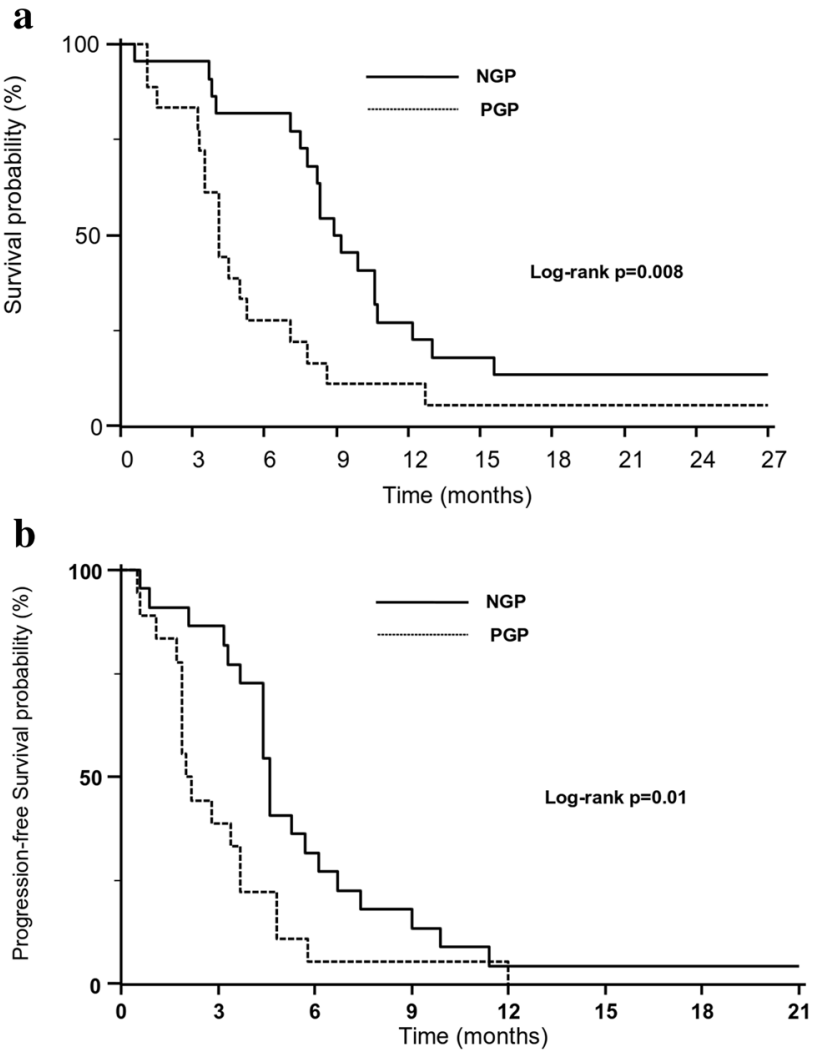

Fig. 4 Results of survival analyses by PGP and NGP status in the 40 patients. Kaplan-Meyer survival curves of overall survival (Panel A) and progression-free survival (Panel B) to second-line paclitaxelramucirumab

In the global analysis of mRNA levels between PT tissues and NM, enhanced expression of GLUT-1, PKM-2, LDH-A and $H K-2$ was found. Except for $H K-1$, these data parallel our findings in colorectal cancer [6]. The variable result of $H K-1$ expression analysis in cancer tissues and the lack of global up-regulation in this study is not surprising. $H K-2$ was found to be overexpressed more than $H K-1$ in several cancer types compared with their normal counterpart [42]. The four hexokinase isoenzymes (HK-1, HK-2, HK-3, and glucokinase) are structurally similar, but only HK-1 and HK-2 are functionally similar. Since hexokinase serves as the gateway through which glucose enters the alternative metabolic pathway, $H K-1$ is redundant to the primary catalytic role of HK-2 to ensure the cancer cell a constant glycolytic flux [43].

According to our criteria, 18 (45\%) primary gastric adenocarcinomas were categorized as having a positive glycolytic profile and, therefore, displaying an intrinsic capability of metabolic adaptation in an unfavorable, hypoxic microenvironment. In fact, the PR combination is not a "pure" antiangiogenic treatment, but it has been demonstrated that even paclitaxel exploits anti-angiogenic mechanisms of action, especially in fractionated regimens [44]. These features may explain why the exposure of these patients to an anti-angiogenic therapy with ramucirumab and paclitaxel produced significantly shorter OS and PFS than patients with a negative glycolytic profile. More than providing novel prognostic information, mediators of an up-regulated glycolytic status may represent the target of novel compounds with tumor metabolism interference activity [45]. Inhibitors of glucose transporters, PKM-2 and LDH-A, attenuate aerobic glycolysis and tumor proliferation with the potential therapeutic role [46], alone or in combination with anti-angiogenic [47] and immune checkpoint [48] therapies.

Unlike our previous analysis in colorectal cancer [6], we did not evaluate $R A S$ mutations. This choice was made considering the low frequency of $R A S$ mutations in gastric cancer (roughly 1-10\%) and the presence instead of $R A S$ amplification [49]. Notably, RAS mutations and RAS amplification may display different oncogenic effects in molecular pathways [49], thus, making it even more difficult to interpret the role of oncogenic $R A S$ in gastric adenocarcinoma. Conversely, we attempted an exploration between glycolytic status with PGP/NGP categories and TP53 mutations. This analysis was supported by high frequency of TP53 mutations in gastric adenocarcinomas $[18,19]$ and the increasing amount of pre-clinical and experimental data, which support a major role of the tumor suppressor gene in orchestrating the glycolytic capability of cancer cells [15, 17]. TP53 mutations were almost equally distributed between PGP and NGP, without any significant association even after considering their sub-classification into disruptive, non-disruptive and gain-of-function.Proof of TP53 driving the Warburg effect mainly derive from pre-clinical studies in cellular and murine models $[15,17]$. It is likely that glycolytic properties of cancer cells in vivo undergo modulation from multiple effectors [50]. Therefore, the putative influence of p53 perturbation is diluted among the several factors that may impact the glycolysis capabilities of cancer cells.

Limitations of this study are its retrospective nature and small sample size. However, our sophisticated analysis of glycolysis profiling in gastric adenocarcinomas, which required primary tumor tissues and the normal mucosa, lays a foundation for future study. This is especially important since no affordable predictive marker of response and survival under the ramucirumab-paclitaxel regimen has been identified [51] and second-line therapy is offered to all patients who may benefit according to clinical criteria.

In conclusion, glycolytic proficiency was found to be associated with adverse survival outcomes of metastatic gastric cancer patients treated with PR systemic therapy. TP53 mutational status alone does not seem to explain such a metabolic shift. Further investigation is needed to confirm our findings that would promote the development of novel therapeutic strategies against cancer metabolism. 
Table 2 Results of the multivariate model analysis

\begin{tabular}{|c|c|c|c|c|}
\hline \multirow[b]{2}{*}{ Variable } & \multicolumn{2}{|l|}{ Overall survival } & \multicolumn{2}{|l|}{ Progression-free Survival } \\
\hline & $\begin{array}{l}\text { HR (95\% confi- } \\
\text { dence interval) }\end{array}$ & $p$ value & HR ( $95 \%$ confidence interval) & $p$ value \\
\hline \multicolumn{5}{|l|}{ Age } \\
\hline$>70$ years vs. $<70$ years & $0.51(0.21-1.16)$ & 0.1 & $0.64(0.29-1.39)$ & 0.2 \\
\hline \multicolumn{5}{|l|}{ Gender } \\
\hline Female vs, Male & $0.53(0.19-1.46$ & 0.5 & $0.48(0.53-3.78)$ & 0.4 \\
\hline \multicolumn{5}{|l|}{ First PFS time } \\
\hline$<6$ months vs, $>6$ months & $2.41(0.92-6.33$ & 0.07 & $1.38(0.60-3.16)$ & 0.4 \\
\hline \multicolumn{5}{|l|}{ Number of metastatic sites } \\
\hline$>2$ vs. 1 & $1.27(0.55-2.96)$ & 0.5 & $1.32(0.59-2.95)$ & 0.5 \\
\hline \multicolumn{5}{|l|}{ Peritoneum involvement } \\
\hline Positive vs. Negative & $2.94(1.21-7.13)$ & 0.01 & $1.42(0.60-3.35)$ & 0.4 \\
\hline \multicolumn{5}{|l|}{ ECOG PS } \\
\hline $1-2$ vs: 0 & $2.56(1.18-5.56)$ & 0.01 & $1.83(0.88-3.79)$ & 0.1 \\
\hline \multicolumn{5}{|l|}{ Lauren's histology } \\
\hline Intestinal vs. Diffuse & $0.46(0.16-1.33)$ & 0.4 & $0.64(0.26-1.54)$ & 0.3 \\
\hline \multicolumn{5}{|l|}{ Grading } \\
\hline 3 vs. $1-2$ & $1.43(0.46-4.43)$ & 0.5 & $1.58(0.48-5.10)$ & 0.4 \\
\hline \multicolumn{5}{|l|}{ Primary tumor resected } \\
\hline No vs. Yes & $3.11(1.03-9.38)$ & 0.04 & $3.39(1.05-10.09)$ & 0.04 \\
\hline \multicolumn{5}{|l|}{ Primary tumor site } \\
\hline Cardia vs. non-cardia & $2.55(0.79-8.26)$ & 0.1 & $1.81(0.66-5.39)$ & 0.4 \\
\hline \multicolumn{5}{|l|}{ Glycolytic status } \\
\hline PGP vs. NGP & $2.57(1.17-5.63)$ & 0.01 & $2.49(1.16-5.38)$ & 0.01 \\
\hline
\end{tabular}

$H R$ hazard ratio, $P G P$ positive glycolytic profile, $N G P$ negative glycolytic profile, $P F S$ progression-free survival, ECOG PS Eastern Cooperative Group Performance Status
Acknowledgements We would like to thank Prof. Cathie Spino (University of Michigan) who reviewed the manuscript and provided editorial assistance. This study has been financially supported by FanoAteneo, Italy.

\section{Compliance with ethical standards}

Conflict of interest The authors declare that they have no conflict of interest.

Ethical approval The study was approved by the "Comitato Etico Regionale delle Marche (CERM)" (Regional Ethic Committee). Italy.

Open Access This article is licensed under a Creative Commons Attribution 4.0 International License, which permits use, sharing, adaptation, distribution and reproduction in any medium or format, as long as you give appropriate credit to the original author(s) and the source, provide a link to the Creative Commons licence, and indicate if changes were made. The images or other third party material in this article are included in the article's Creative Commons licence, unless indicated otherwise in a credit line to the material. If material is not included in the article's Creative Commons licence and your intended use is not permitted by statutory regulation or exceeds the permitted use, you will need to obtain permission directly from the copyright holder. To view a copy of this licence, visit http://creativecommons.org/licenses/by/4.0/.

\section{References}

1. Ngo DC, Ververis K, Tortorella SM, Karagiannis TC. Introduction to the molecular basis of cancer metabolism and the Warburg effect. Mol Biol Rep. 2015;42:819-23.

2. Cantor JR, Sabatini DM. Cancer cell metabolism: one hallmark, many faces. Cancer Discov. 2012;2:881-98.

3. Feng Y, Xiong Y, Qiao T, Li X, Jia L, Han Y. Lactate dehydrogenase A: a key player in carcinogenesis and potential target in cancer therapy. Cancer Med. 2018;7:6124-36.

4. Herling A, König M, Bulik S, Holzhütter HG. Enzymatic features of the glucose metabolism in tumor cells. FEBS J. 2011;278:2436-59.

5. Chaneton B, Gottlieb E. Rocking cell metabolism: revised functions of the key glycolytic regulator PKM2 in cancer. Trends Biochem Sci. 2012;37:309-16.

6. Graziano F, Ruzzo A, Giacomini E, Ricciardi T, Aprile G, Loupakis F, et al. Glycolysis gene expression analysis and selective metabolic advantage in the clinical progression of colorectal cancer. Pharmacogenomics J. 2017;17:258-64.

7. Jiménez-Valerio G, Casanovas O. Angiogenesis and metabolism: entwined for therapy resistance. Trends Cancer. 2017;3:10-8.

8. Abdullah SE, Perez-Soler R. Mechanisms of resistance to vascular endothelial growth factor blockade. Cancer. 2012;118:3455-67.

9. Itatani Y, Kawada K, Yamamoto T, Sakai Y. Resistance to antiangiogenic therapy in cancer-alterations to anti-VEGF pathway. Int J Mol Sci. 2018;19(4):E1232. 
10. Wilde L, Roche M, Domingo-Vidal M, Tanson K, Philip N, Curry J, Martinez-Outschoorn U. Metabolic coupling and the Reverse Warburg Effect in cancer: Implications for novel biomarker and anticancer agent development. Semin Oncol. 2017;44:198-203.

11. Paolicchi E, Gemignani F, Krstic-Demonacos M, Dedhar S, Mutti L, Landi S. Targeting hypoxic response for cancer therapy. Oncotarget. 2016;7:13464-78.

12. Lord S, Funes JM, Harris AL, Quintela-Fandino M. Antiangiogenic resistance and cancer metabolism: opportunities for synthetic lethality. Curr Drug Targets. 2016;17:1714-27.

13. Abdel-Wahab AF, Mahmoud W, Al-Harizy RM. Targeting glucose metabolism to suppress cancer progression: prospective of anti-glycolytic cancer therapy. Pharmacol Res. 2019;150:104511.

14. Casak SJ, Fashoyin-Aje I, Lemery SJ, Zhang L, Jin R, Li H, et al. FDA approval summary: ramucirumab for gastric cancer. Clin Cancer Res. 2015;21:3372-6.

15. Zhang C, Liu J, Liang Y, Wu R, Zhao Y, Hong X, et al. Tumourassociated mutant p53 drives the Warburg effect. Nat Commun. 2013;4:2935

16. Eriksson M, Ambroise G, Ouchida AT, Lima Queiroz A, Smith D, Gimenez-Cassina A et al. Effect of mutant p53 proteins on glycolysis and mitochondrial metabolism. Mol Cell Biol. 2017;37(24) e00328-17.

17. Itahana $\mathrm{Y}$, Itahana K. Emerging Roles of p53 Family Members in Glucose Metabolism. Int J Mol Sci. 2018;19.

18. Sabapathy K, Lane DP. Therapeutic targeting of p53: all mutants are equal, but some mutants are more equal than others. Nat Rev Clin Oncol. 2018;15:13-30.

19. Muller PA, Vousden KH. Mutant p53 in cancer: new functions and therapeutic opportunities. Cancer Cell. 2014;25:304-17.

20. Di Bartolomeo M, Niger M, Tirino G, Petrillo A, Berenato R, Laterza MM, et al. Ramucirumab as second-line therapy in metastatic gastric cancer: real-world data from the RAMoss study. Target Oncol. 2018;13(2):227-34.

21. Guescini M, Sisti D, Rocchi MB, Stocchi L, Stocchi V. A new real-time PCR method to overcome significant quantitative inaccuracy due to slight amplification inhibition. BMC Bioinformatics. 2008;9:326.

22. Livak KJ, Schmittgen TD. Analysis of relative gene expression data using real-time quantitative PCR and the 2(-Delta Delta C(T) Method. Methods. 2001;25:402-8.

23. Shi L, Jones WD, Jensen RV, Harris SC, Perkins RG, Goodsaid FM, et al. The balance of reproducibility, sensitivity, and specificity of lists of differentially expressed genes in microarray studies. BMC Bioinformatics. 2008;9:S10.

24. Petersen D, Chandramouli GV, Geoghegan J, Hilburn J, Paarlberg J, Kim CH, et al. Three microarray platforms: an analysis of their concordance in profiling gene expression. BMC Genomics. 2005;6:63.

25. Hariparsad N, Ramsden D, Palamanda J, Dekeyser JG, Fahmi OA, Kenny JR, et al. Considerations from the IQ induction working group in response to drug-drug interaction guidance from regulatory agencies: focus on downregulation, CYP2C induction, and CYP2B6 positive control. Drug Metab Dispos. 2017;45:1049-59.

26. Robinson JT, Thorvaldsdóttir H, Wenger AM, Zehir A, Jill P. Variant review with the integrative genomics viewer (IGV). Cancer Res. 2017;77:e31-e3434.

27. Poeta ML, Manola J, Goldwasser MA, Forastiere A, Benoit $\mathrm{N}$, Califano JA, et al. TP53 mutations and survival in squamous-cell carcinoma of the head and neck. N Engl J Med. 2007;357:2552-611.

28. Yu M, Chen S, Hong W, Gu Y, Huang B, Lin Y, et al. Prognostic role of glycolysis for cancer outcome: evidence from 86 studies. J Cancer Res Clin Oncol. 2019;145:967-99.
29. Berlth F, Mönig S, Pinther B, Grimminger P, Maus M, Schlösser $\mathrm{H}$, et al. Both GLUT-1 and GLUT-14 are independent prognostic factors in gastric adenocarcinoma. Ann Surg Oncol. 2015;22(Suppl 3):S822-S831831.

30. Kawamura T, Kusakabe T, Sugino T, Watanabe K, Fukuda T, Nashimoto A, et al. Expression of glucose transporter-1 in human gastric carcinoma: association with tumor aggressiveness, metastasis, and patient survival. Cancer. 2001;92:634-41.

31. Schlößer HA, Drebber U, Urbanski A, Haase S, Baltin C, Berlth F, et al. Glucose transporters 1, 3, 6, and 10 are expressed in gastric cancer and glucose transporter 3 is associated with UICC stage and survival. Gastric Cancer. 2017;20:83-91.

32. Qiu MZ, Han B, Luo HY, Zhou ZW, Wang ZQ, Wang FH, et al. Expressions of hypoxia-inducible factor- $1 \alpha$ and hexokinase-II in gastric adenocarcinoma: the impact on prognosis and correlation to clinicopathologic features. Tumour Biol. 2011;32:159-66.

33. Rho M, Kim J, Jee CD, Lee YM, Lee HE, Kim MA, et al. Expression of type 2 hexokinase and mitochondria-related genes in gastric carcinoma tissues and cell lines. Anticancer Res. 2007;27:251-8.

34. Gao Y, Xu D, Yu G, Liang J. Overexpression of metabolic markers HK1 and PKM2 contributes to lymphatic metastasis and adverse prognosis in Chinese gastric cancer. Int J Clin Exp Pathol. 2015;8:9264-71.

35. Lim JY, Yoon SO, Seol SY, Hong SW, Kim JW, Choi SH, et al. Overexpression of the M2 isoform of pyruvate kinase is an adverse prognostic factor for signet ring cell gastric cancer. World J Gastroenterol. 2012;18:4037-43.

36. Wang J, Yuan W, Chen Z, Wu S, Chen J, Ge J, et al. Overexpression of G6PD is associated with poor clinical outcome in gastric cancer. Tumour Biol. 2012;33:95-101.

37. Kim JG, Lee SJ, Chae YS, Kang BW, Lee YJ, Oh SY, et al. Association between phosphorylated AMP-activated protein kinase and MAPK3/1 expression and prognosis for patients with gastric cancer. Oncology. 2013;85:78-85.

38. Sun X, Sun Z, Zhu Z, Li C, Zhang J, Xu H, et al. Expression of SIP1 is strongly correlated with LDHA and shows a significantly poor outcome in gastric cancer. Tumour Biol. 2015;36:7521-30.

39. Kim HS, Lee HE, Yang HK, Kim WH. High lactate dehydrogenase 5 expressioncorrelates with high tumoral and stromal vascular endothelial growth factor expression in gastric cancer. Pathobiology. 2014;81:78-85.

40. Kolev Y, Uetake H, Takagi Y, Sugihara K. Lactate dehydrogenase-5 (LDH-5) expression in human gastric cancer: association with hypoxia-inducible factor (HIF-1alpha) pathway, angiogenic factors production and poor prognosis. Ann Surg Oncol. 2008; $15: 2336-444$

41. TeSlaa T, Teitell MA. Techniques to monitor glycolysis. Methods Enzymol. 2014;542:91-114.

42. Smith TA. Mammalian hexokinases and their abnormal expression in cancer. Br J Biomed Sci. 2000;57:170-8.

43. Wilson JE. Isozymes of mammalian hexokinase: structure, subcellular localization and metabolic function. J Exp Biol. 2003;206:2049-57.

44. Bocci G, Di Paolo A, Danesi R. The pharmacological bases of the antiangiogenic activity of paclitaxel. Angiogenesis. 2013;16:481-92.

45. El Hassouni B, Granchi C, Vallés-Martí A, Supadmanaba IGP, Bononi G, Tuccinardi T, et al. The dichotomous role of the glycolytic metabolism pathway in cancer metastasis: Interplay with the complex tumor microenvironment and novel therapeutic strategies. Semin Cancer Biol. 2020;60:238-248.

46. Mohammad GH, Vassileva V, Acedo P, Olde Damink SWM, Malago M, Dhar DK, et al. Targeting pyruvate kinase M2 and lactate dehydrogenase $\mathrm{A}$ is an effective combination strategy for the treatment of pancreatic cancer. Cancers (Basel). 2019;11:E1372. 
47. Pisarsky L, Bill R, Fagiani E, Dimeloe S, Goosen RW, Hagmann $\mathrm{J}$, et al. Targeting metabolic symbiosis to overcome resistance to 33 anti-angiogenic therapy. Cell Rep. 2016;15:1161-74.

48. Renner K, Bruss C, Schnell A, Koehl G, Becker HM, Fante M, et al. Restricting glycolysis preserves $\mathrm{T}$ cell effector functions and augments checkpoint therapy. Cell Rep. 2019;29(135-150):e9.

49. Wong GS, Zhou J, Liu JB, Wu Z, Xu X, Li T, et al. Targeting wildtype KRAS-amplified gastroesophageal cancer through combined MEK and SHP2 inhibition. Nat Med. 2018;24:968-77.

50. Liu Y, Zhang Z, Wang J, Chen C, Tang X, Zhu J, et al. Metabolic reprogramming results in abnormal glycolysis in gastric cancer: a review. Onco Targets Ther. 2019;12:1195-204.
51. Van Cutsem E, Muro K, Cunningham D, Bodoky G, Sobrero A, Cascinu $\mathrm{S}$, et al. RAINBOW Investigators Biomarker analyses of second-line ramucirumab in patients with advanced gastric cancer from RAINBOW, a global, randomized, double-blind, phase 3 study. Eur J Cancer. 2020;127:150-7.

Publisher's Note Springer Nature remains neutral with regard to jurisdictional claims in published maps and institutional affiliations.

\section{Affiliations}

\section{Annamaria Ruzzo ${ }^{1}$ (D) . Francesco Graziano ${ }^{2}$. Irene Bagaloni ${ }^{1} \cdot$ Maria Di Bartolomeo $^{3} \cdot$ Michele Prisciandaro $^{3}$. Giuseppe Aprile ${ }^{4}$ Elena Ongaro ${ }^{5}$ - Bruno Vincenzi ${ }^{6}$. Giuseppe Perrone ${ }^{6}$. Daniele Santini ${ }^{6}$. Lorenzo Fornaro ${ }^{7}$. Caterina Vivaldi ${ }^{7}$. Gianluca Tomasello ${ }^{8}$. Fotios Loupakis ${ }^{9} \cdot$ Sara Lonardi $^{9} \cdot$ Matteo Fassan $^{10} \cdot$ Michele Valmasoni $^{11}$. Donatella Sarti ${ }^{2} \cdot$ Paola Lorenzini ${ }^{2} \cdot$ Vincenzo Catalano $^{2} \cdot$ Renato Bisonni $^{12} \cdot$ Michela Del Prete $^{12} \cdot$ Guido Collina $^{13}$. Mauro Magnani ${ }^{1}$}

1 Department of Biomolecular Sciences (DiSB), University of Urbino "Carlo Bo", Via Arco d'Augusto, 2, 61032 Fano, PU, Italy

2 Department of Onco-Hematology, Division of Oncology, Azienda Ospedaliera "Ospedali Riuniti Marche Nord", 61122 Pesaro, Italy

3 Oncologia Medica GI Fondazione IRCCS Istituto Tumori Milano, Milan, Italy

4 Department of Medical Oncology, San Bortolo General Hospital, AULSS8 Berica, Vicenza, Italy

5 Department of Oncology, University and General Hospital, Udine, Italy

6 University Campus Bio-Medico, Rome, Italy

7 Azienda Ospedaliero-Universitaria Pisana, Pisa, Italy
8 S. C. Oncologia, ASST Hospital of Cremona, Cremona, Italy

9 Department of Oncology, Veneto Institute of Oncology IOV - IRCCS, Padua, Italy

10 Surgical Pathology Unit, Department of Medicine (DIMED), University of Padua, Padua, Italy

11 Clinica Chirurgica 3, Department of Surgical, Oncological and Gastroenterological Sciences (DISCOG), University of Padua, Padua, Italy

12 Medical Oncology Unit, Hospital of Fermo, Fermo, Italy

13 Area vasta 5, Ospedale "C. e G. Mazzoni” Ascoli Piceno, Ascoli Piceno, Italy 